\title{
A GLOBAL NULLSTELLENSATZ FOR IDEALS OF DENJOY-CARLEMAN FUNCTIONS
}

\author{
FRANCESCA ACQUISTAPACE, FABRIZIO BROGLIA, AND ANDREEA C. NICOARA
}

\author{
(Communicated by Lev Borisov)
}

\begin{abstract}
We prove a Nullstellensatz result for global ideals of DenjoyCarleman quasi-analytic functions in both finitely generated and infinitely generated cases.
\end{abstract}

\section{INTRODUCTION}

Let $\mathfrak{a}$ be an ideal in a ring $\mathcal{C}\left(\mathbb{R}^{n}\right)$ of Denjoy-Carleman quasi-analytic functions so that the process of resolution of singularities applies to $\mathcal{C}\left(\mathbb{R}^{n}\right)$; see [3] and [4]. The classical theory of the Denjoy-Carleman quasi-analytic functions may be found in [5], while [6] is a comprehensive recent survey. We will characterize here the ideal $\mathcal{I}(\mathcal{Z}(\mathfrak{a}))$ of functions vanishing on the variety of the ideal $\mathfrak{a}$ in terms of the Łojasiewicz radical, another notion of Łojasiewicz radical computed on compact sets, and the saturation.

1.1. Theorem. Let $\mathfrak{a}$ be an ideal in $\mathcal{C}\left(\mathbb{R}^{n}\right)$.

(i) If $\mathfrak{a}$ is finitely generated, then $\mathcal{I}(\mathcal{Z}(\mathfrak{a}))=\widetilde{E} \mathfrak{a}$, the saturation of the Eojasiewicz radical of $\mathfrak{a}$.

(ii) $\mathcal{I}(\mathcal{Z}(\mathfrak{a}))=(\sqrt[E]{\mathfrak{a}})_{K}$, where $(\sqrt[E]{\mathfrak{a}})_{K}$ is the Lojasiewicz radical of $\mathfrak{a}$ computed on all compact subsets of $\mathbb{R}^{n}$.

The main theorem will be derived from the following result proven in [2].

1.2. Theorem. Let $\mathfrak{a} \subset \mathcal{E}\left(\mathbb{R}^{n}\right)$ be a Eojasiewicz ideal, where $\mathcal{E}\left(\mathbb{R}^{n}\right)$ is the ring of smooth functions on $\mathbb{R}^{n}$. Then the following hold:

(a) $\widetilde{E} \mathfrak{a}=\overline{\sqrt[t]{\mathfrak{a}}}$, i.e. the saturation of $\sqrt[t]{\mathfrak{a}}$ equals its Whitney closure.

(b) $\mathcal{I}(\mathcal{Z}(\mathfrak{a}))=\overline{\sqrt[t]{\mathfrak{a}}}$.

Received by the editors December 31, 2014 and, in revised form, April 25, 2015 and May 28, 2015 .

2010 Mathematics Subject Classification. Primary 26E10, 46E25; Secondary 11E25.

Key words and phrases. Denjoy-Carleman quasianalytic classes, ideals of Denjoy-Carleman functions, Nullstellensatz, closed ideal, real Nullstellensatz, radical ideal, Łojasiewicz radical ideal, saturation of an ideal.

The work presented in this paper was initiated while the authors were visiting the American Institute of Mathematics, Palo Alto, CA, for a SQuaRE workshop. The authors are grateful to AIM for the hospitality and financial support. 


\section{Definitions}

We start by defining $\mathcal{C}\left(\mathbb{R}^{n}\right)$ in the most general way such that it contains all real-analytic functions on $\mathbb{R}^{n}$ and the resolution of singularities holds for finitely generated ideals in $\mathcal{C}\left(\mathbb{R}^{n}\right)$; see [3].

2.1. Definition. $\mathcal{C}\left(\mathbb{R}^{n}\right)$ is an $\mathbb{R}$-subalgebra of $\mathcal{E}\left(\mathbb{R}^{n}\right)$ such that on every open set $U$ in $\mathbb{R}^{n}$, the following properties hold:

(i) $\mathcal{O}(U) \subset \mathcal{C}(U)$, where $\mathcal{O}(U)$ denotes the algebra of restrictions to $U$ of realanalytic functions on $\mathbb{R}^{n}$.

(ii) $\mathcal{C}$ is closed under composition with mappings whose components are in $\mathcal{C}$.

(iii) $\mathcal{C}$ is closed under differentiation.

(iv) $\mathcal{C}$ is quasi-analytic, namely if $f \in \mathcal{C}(U), a \in U$, and the Taylor series of $f$ at $a$ is identically zero, $T_{a} f \equiv 0$, then $f$ vanishes in a neighborhood of $a$.

(v) $\mathcal{C}$ is closed under division by a coordinate.

(vi) $\mathcal{C}$ is closed under inverse, and hence it satisfies the Implicit Function Theorem.

The statements of Theorems 1.1 and 1.2 contain the saturation, the Whitney closure, two notions of Łojasiewicz radical, as well as the notion of a Lojasiewicz ideal. We define all of them now.

2.2. Definition. The saturation of an ideal $\mathfrak{a}$ in $\mathcal{C}\left(\mathbb{R}^{n}\right)$ is the ideal

$$
\tilde{\mathfrak{a}}=\left\{g \in \mathcal{C}\left(\mathbb{R}^{n}\right) \mid \forall x \in \mathbb{R}^{n} g_{x} \in \mathfrak{a C _ { x }}\right\},
$$

where $\mathcal{C}_{x}$ is the local ring of germs of Denjoy-Carleman functions at the point $x$. The saturation of an ideal $\mathfrak{a}$ in $\mathcal{E}\left(\mathbb{R}^{n}\right)$ is the ideal

$$
\tilde{\mathfrak{a}}=\left\{g \in \mathcal{E}\left(\mathbb{R}^{n}\right) \mid \forall x \in \mathbb{R}^{n} g_{x} \in \mathfrak{a} \mathcal{E}_{x}\right\},
$$

where $\mathcal{E}_{x}$ is the ring of germs of smooth functions in $n$ variables at $x$.

As shown in [2], if $\mathfrak{a}$ is an ideal in $\mathcal{E}\left(\mathbb{R}^{n}\right)$, then

$$
\begin{aligned}
\tilde{\mathfrak{a}}= & \left\{g \in \mathcal{E}\left(\mathbb{R}^{n}\right) \mid \forall \text { compact } K \subset \mathbb{R}^{n} \exists h \in \mathcal{E}\left(\mathbb{R}^{n}\right)\right. \text { s.t. } \\
& \mathcal{Z}(h) \cap K=\emptyset \text { and } h g \in \mathfrak{a}\} \\
= & \left\{g \in \mathcal{E}\left(\mathbb{R}^{n}\right) \mid \forall x \in \mathbb{R}^{n} \exists h \in \mathcal{E}\left(\mathbb{R}^{n}\right) \text { s.t. } h(x) \neq 0 \text { and } h g \in \mathfrak{a}\right\},
\end{aligned}
$$

where $\mathcal{Z}(h)$ is the zero set of $h$.

2.3. Definition. Let the algebra $\mathcal{E}\left(\mathbb{R}^{n}\right)$ of smooth real-valued functions on $\mathbb{R}^{n}$ be endowed with the compact open topology. Let $\mathfrak{a}$ be an ideal in $\mathcal{E}\left(\mathbb{R}^{n}\right)$. The Whitney closure $\overline{\mathfrak{a}}$ is the closure of $\mathfrak{a}$ in this topology. By the Whitney Spectral Theorem, $\overline{\mathfrak{a}}=\mathfrak{a}^{*}$, where

$$
\mathfrak{a}^{*}=\left\{g \in \mathcal{E}\left(\mathbb{R}^{n}\right) \mid \forall x \in \mathbb{R}^{n} T_{x} g \in T_{x} \mathfrak{a}\right\} .
$$

2.4. Definition. The Eojasiewicz radical of an ideal $\mathfrak{a}$ in $\mathcal{E}\left(\mathbb{R}^{n}\right)$ is given by

$$
\sqrt[\mathfrak{L}]{\mathfrak{a}}:=\left\{g \in \mathcal{E}\left(\mathbb{R}^{n}\right) \mid \exists f \in \mathfrak{a} \text { and } m \geq 1 \text { s.t. } f-g^{2 m} \geq 0 \text { on } \mathbb{R}^{n}\right\} .
$$

If $\mathfrak{a}$ is an ideal in $\mathcal{C}\left(\mathbb{R}^{n}\right)$, then

$$
\sqrt[\mathfrak{t}]{\mathfrak{a}}:=\left\{g \in \mathcal{C}\left(\mathbb{R}^{n}\right) \mid \exists f \in \mathfrak{a} \text { and } m \geq 1 \text { s.t. } f-g^{2 m} \geq 0 \text { on } \mathbb{R}^{n}\right\} .
$$

2.5. Definition. The Eojasiewicz radical computed on all compact subsets of $\mathbb{R}^{n}$ of an ideal $\mathfrak{a}$ in $\mathcal{C}\left(\mathbb{R}^{n}\right)$ is given by

$$
\begin{array}{r}
(\sqrt[\mathrm{t}]{\mathfrak{a}})_{K}:=\left\{g \in \mathcal{C}\left(\mathbb{R}^{n}\right) \mid \forall \text { compact } K \subset \mathbb{R}^{n} \exists f \in \mathfrak{a} \text { and } m \geq 1\right. \\
\text { s.t. } \left.f-g^{2 m} \geq 0 \text { on } K\right\} .
\end{array}
$$


2.6. Definition. An ideal $\mathfrak{a} \subset \mathcal{E}\left(\mathbb{R}^{n}\right)$ is a Eojasiewicz ideal if

(1) $\mathfrak{a}$ is generated by finitely many smooth functions $f_{1}, \ldots, f_{l}$;

(2) $\mathfrak{a}$ contains an element $f$ with the property that for any compact $K \subset \mathbb{R}^{n}$ there exist a constant $c$ and an integer $m$ such that $|f(x)| \geq c d(x, \mathcal{Z}(\mathfrak{a}))^{m}$ on an open neighborhood of $K$.

2.7. Remark. The element $f$ can be taken to be the sum of squares of the generators $f_{1}^{2}+\cdots+f_{l}^{2}$.

\section{Proof of Theorem 1.1}

Proof of part (i) of Theorem 1.1. The ideal $\mathfrak{a}$ is finitely generated so $\mathfrak{a}=\left(f_{1}, \ldots, f_{l}\right)$. In 3 Bierstone and Milman extended the resolution of singularities to the DenjoyCarleman quasi-analytic classes. As a result, each of the generators $f_{i}$ of $\mathfrak{a}$ satisfies a Łojasiewicz inequality; see Theorem 6.3 of $\left[3\right.$. Let $\hat{\mathfrak{a}}$ be the ideal in the $\operatorname{ring} \mathcal{E}\left(\mathbb{R}^{n}\right)$ generated by the elements of $\mathfrak{a}$. Clearly, $\hat{\mathfrak{a}}$ is a Łojasiewicz ideal since $f=f_{1}^{2}+\cdots+f_{l}^{2}$ satisfies part (2) of Definition 2.6. Theorem 1.2 then implies that $\mathcal{I}(\mathcal{Z}(\hat{\mathfrak{a}}))=\sqrt[\mathbb{E}]{\hat{\mathfrak{a}}}$. Since $\mathcal{Z}(\mathfrak{a})=\mathcal{Z}(\hat{\mathfrak{a}})$, it follows that $\mathcal{I}(\mathcal{Z}(\mathfrak{a}))=\mathcal{I}(\mathcal{Z}(\hat{\mathfrak{a}})) \cap \mathcal{C}\left(\mathbb{R}^{n}\right)=\widetilde{\sqrt[\mathrm{E}]{\hat{\mathfrak{a}}}} \cap \mathcal{C}\left(\mathbb{R}^{n}\right)$. If we

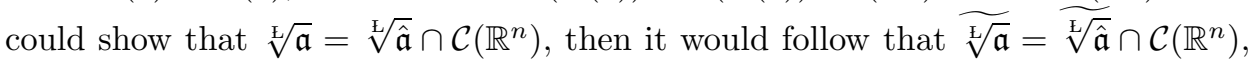
where saturation on the left-hand side is in $\mathcal{C}\left(\mathbb{R}^{n}\right)$, while saturation on the righthand side is in $\mathcal{E}\left(\mathbb{R}^{n}\right)$. $\sqrt[\mathrm{L}]{\mathfrak{a}}=\sqrt[\mathrm{L}]{\hat{\mathfrak{a}}} \cap \mathcal{C}\left(\mathbb{R}^{n}\right)$ is a consequence of the following lemma.

3.1. Lemma. Let $\mathcal{O}\left(\mathbb{R}^{n}\right)$ be the ring of real-analytic functions on $\mathbb{R}^{n}$, and let $\mathcal{A}\left(\mathbb{R}^{n}\right)$ be any ring of functions on $\mathbb{R}^{n}$ such that $\mathcal{O}\left(\mathbb{R}^{n}\right) \subset \mathcal{A}\left(\mathbb{R}^{n}\right) \subset \mathcal{E}\left(\mathbb{R}^{n}\right)$. If $\mathfrak{a}$ is any ideal in $\mathcal{A}\left(\mathbb{R}^{n}\right)$ and if $\hat{\mathfrak{a}}$ is the ideal generated by the elements of $\mathfrak{a}$ in $\mathcal{E}\left(\mathbb{R}^{n}\right)$, then $\sqrt[E]{\mathfrak{a}}=\sqrt[E]{\hat{\mathfrak{a}}} \cap \mathcal{A}\left(\mathbb{R}^{n}\right)$.

3.2. Remark. It is not necessary for $\mathfrak{a}$ to be finitely generated.

Proof. Note that $\sqrt[\mathrm{t}]{\mathfrak{a}} \subset \sqrt[\mathrm{t}]{\hat{\mathfrak{a}}} \cap \mathcal{A}\left(\mathbb{R}^{n}\right)$ is trivially true, so the proof of the lemma reduces to showing the reverse inequality $\sqrt[\mathrm{E}]{\hat{\mathfrak{a}}} \cap \mathcal{A}\left(\mathbb{R}^{n}\right) \subset \sqrt[\mathrm{t}]{\mathfrak{a}}$.

Let $g \in \sqrt[\mathrm{E}]{\hat{\mathfrak{a}}} \cap \mathcal{A}\left(\mathbb{R}^{n}\right)$, then there exist $f \in \hat{\mathfrak{a}}$ and $m \geq 1$ such that $f \geq g^{2 m}$ on $\mathbb{R}^{n}$. The latter implies $f^{2} \geq g^{4 m}$ on $\mathbb{R}^{n}$. Since $f \in \hat{\mathfrak{a}}$, there exist $f_{1}, \ldots, f_{k} \in \mathfrak{a}$, $h_{1}, \ldots, h_{k} \in \mathcal{E}\left(\mathbb{R}^{n}\right)$ such that $f=h_{1} f_{1}+\cdots+h_{k} f_{k}$. Therefore, $|f| \leq\left|h_{1}\right|\left|f_{1}\right|+$ $\cdots+\left|h_{k}\right|\left|f_{k}\right|$, so

$$
|f|^{2} \leq\left(\left|h_{1}\right|\left|f_{1}\right|+\cdots+\left|h_{k}\right|\left|f_{k}\right|\right)^{2} \leq k\left(\left|h_{1}\right|^{2}\left|f_{1}\right|^{2}+\cdots+\left|h_{k}\right|^{2}\left|f_{k}\right|^{2}\right) .
$$

If we could bound from above the coefficient functions $h_{1}, \ldots, h_{k}$ by functions in $\mathcal{A}\left(\mathbb{R}^{n}\right)$, we would be done. Let $\epsilon>0$ be given. Since the coefficient functions are smooth, they are clearly continuous, so there exist global real-analytic functions $\tilde{h}_{1}, \ldots, \tilde{h}_{k} \in \mathcal{O}\left(\mathbb{R}^{n}\right)$ such that $\left|\tilde{h}_{i}(x)-h_{i}(x)\right|<\epsilon$ for all $x \in \mathbb{R}^{n}$ and all $i=1, \ldots, k$. Therefore, $h_{i}(x)<\tilde{h}_{i}(x)+\epsilon$ for all $x \in \mathbb{R}^{n}$ and all $i=1, \ldots, k$. Note that $\mathcal{O}\left(\mathbb{R}^{n}\right) \subset$ $\mathcal{A}\left(\mathbb{R}^{n}\right)$. We have thus shown that

$$
g^{4 m} \leq f^{2}<k\left(\left(\tilde{h}_{1}^{2}+\epsilon\right)^{2} f_{1}^{2}+\cdots+\left(\tilde{h}_{k}^{2}+\epsilon\right)^{2} f_{k}^{2}\right),
$$

where $\tilde{f}=k\left(\left(\tilde{h}_{1}^{2}+\epsilon\right)^{2} f_{1}^{2}+\cdots+\left(\tilde{h}_{k}^{2}+\epsilon\right)^{2} f_{k}^{2}\right) \in \mathfrak{a}$ as needed.

We now let $\mathcal{A}\left(\mathbb{R}^{n}\right)=\mathcal{C}\left(\mathbb{R}^{n}\right)$ noting that global real-analytic functions are contained in any Denjoy-Carleman ring of global functions of the type we are considering. We obtain $\sqrt[\mathrm{E}]{\mathfrak{a}}=\sqrt[\mathrm{E}]{\hat{\mathfrak{a}}} \cap \mathcal{C}\left(\mathbb{R}^{n}\right)$, hence $\widetilde{\sqrt[E]{\mathfrak{a}}}=\widetilde{\sqrt[E]{\hat{\mathfrak{a}}}} \cap \mathcal{C}\left(\mathbb{R}^{n}\right)$. 
3.3. Remarks. (1) It should be noted here that part (i) of Theorem 1.1 parallels the result obtained by Acquistapace, Broglia, and Fernando in [1] for an ideal of global real-analytic functions on a $C$-analytic set.

(2) Let $\mathcal{A}\left(\mathbb{R}^{n}\right)$ be a non-quasi-analytic class containing all real-analytic functions. Let $\mathfrak{a}$ be a finitely generated ideal in $\mathcal{A}\left(\mathbb{R}^{n}\right)$ such that the definition of a Łojasiewicz ideal, Definition 2.6. is verified for $\hat{\mathfrak{a}}$. The proof given above shows that part (i) of Theorem 1.1 holds for $\mathfrak{a}$. Characterizing which non-quasi-analytic functions satisfy Łojasiewicz inequalities is an open problem, however. Only isolated examples were given by the Malgrange school.

Proof of part (ii) of Theorem 1.1. The ideal a here is not necessarily finitely generated. Note that only $\mathcal{I}(\mathcal{Z}(\mathfrak{a})) \subset(\sqrt[\mathrm{E}]{\mathfrak{a}})_{K}$ needs to be proven as $(\sqrt[\mathrm{L}]{\mathfrak{a}})_{K} \subset \mathcal{I}(\mathcal{Z}(\mathfrak{a}))$ is obvious. Consider $g \in \mathcal{I}(\mathcal{Z}(\mathfrak{a}))$. Let $K$ be any compact subset of $\mathbb{R}^{n}$. We will use topological noetherianity, a consequence of the resolution of singularities for the Denjoy-Carleman classes, to show there exist a finite number of elements of the original ideal $f_{1}, \ldots, f_{l} \in \mathfrak{a}$ and an open set $U \supset K$ such that $\mathcal{Z}(\mathfrak{a}) \cap U=\mathcal{Z}\left(\left(f_{1}, \ldots, f_{l}\right)\right) \cap U$. If $\mathfrak{a}=(0)$, there is nothing to be proven, so assume $\mathfrak{a} \neq(0)$, and take some $f_{1} \in \mathfrak{a}$ not identically zero. If there exists an open set $U \supset K$ such that $\mathcal{Z}\left(\left(f_{1}\right)\right)=\mathcal{Z}(\mathfrak{a})$ on $U$, we are done with $l=1$; otherwise, there is some $f_{2} \in \mathfrak{a}$ such that $\mathcal{Z}\left(\left(f_{1}, f_{2}\right)\right) \subsetneq \mathcal{Z}\left(\left(f_{1}\right)\right)$. Inductively, we will have chosen $f_{1}, \ldots, f_{k} \in \mathfrak{a}$ such that $\mathcal{Z}\left(\left(f_{1}, f_{2}, \ldots, f_{k}\right)\right) \subsetneq \mathcal{Z}\left(\left(f_{1}, f_{2}, \ldots, f_{k-1}\right)\right)$. Hence we have a sequence of finitely generated ideals $\left(f_{1}\right) \subset\left(f_{1}, f_{2}\right) \subset \ldots \subset\left(f_{1}, \ldots, f_{k}\right) \subset \ldots$

Topological noetherianity, Theorem 6.1 in [3] (see also Theorem 3.1 in [4]), guarantees that the sequence of corresponding zero sets stabilizes, i.e. there exists some $k$ and some open set $U \supset K$ such that $\mathcal{Z}\left(\left(f_{1}, f_{2}, \ldots, f_{k}\right)\right)=\mathcal{Z}(\mathfrak{a})$ on $U$. Set $l=k$. We have obtained $\mathcal{Z}(\mathfrak{a}) \cap U=\mathcal{Z}\left(\left(f_{1}, \ldots, f_{l}\right)\right) \cap U$. We now recall the following lemma from [2].

3.4. Lemma. Let $\mathfrak{a}$ be a Eojasiewicz ideal generated by $f_{1}, \ldots, f_{l}$ and $f=f_{1}^{2}+\cdots+$ $f_{l}^{2}$. Let $g \in \mathcal{E}\left(\mathbb{R}^{n}\right)$ be such that $\mathcal{Z}(g) \supset \mathcal{Z}(f)=\mathcal{Z}(\mathfrak{a})$. Then for any compact set $K \subset \mathbb{R}^{n}$, there exist a constant $c$ and a positive integer $m$ such that $g^{2 m} \leq c f$ on an open neighborhood of $K$. In particular, there exist an integer $m$ and an element $a \in \mathfrak{a}$ such that $g^{2 m} \leq|a|$ on an open neighborhood of $K$.

Its proof from [2] carries over if instead of $\mathbb{R}^{n}$ we restrict to an open set $U \subset \mathbb{R}^{n}$ provided that all compact sets considered are subsets of $U$. The ideal generated by $f_{1}, \ldots, f_{l}$ in $\mathcal{E}\left(\mathbb{R}^{n}\right)$ is Eojasiewicz and since $g \in \mathcal{I}(\mathcal{Z}(\mathfrak{a}))$, it follows that $\left.\mathcal{Z}(g)\right|_{U} \supset$ $\left.\mathcal{Z}\left(\left(f_{1}, \ldots, f_{l}\right)\right)\right|_{U}$. By the lemma, there exist a constant $c$ and a positive integer $m$ such that $g^{2 m} \leq c f$ on some set $\tilde{U}$, where $K \subset \tilde{U} \subset U$. Therefore, $g \in(\sqrt[\mathrm{E}]{\mathfrak{a}})_{K}$.

3.5. Remark. Unlike $\sqrt[\mathrm{L}]{\cdot},(\sqrt[\mathrm{E}]{\cdot})_{K}$ already contains in its definition a saturation operation. In other words, for any ideal $\mathfrak{a}$ in $\mathcal{C}\left(\mathbb{R}^{n}\right),(\sqrt[\mathrm{E}]{\mathfrak{a}})_{K}$ is already saturated, while $\sqrt[\mathfrak{E}]{\mathfrak{a}}$ might not be.

\section{REFERENCES}

[1] Francesca Acquistapace, Fabrizio Broglia, and José F. Fernando, On the Nullstellensätze for Stein Spaces and C-analytic Sets, Preprint. arXiv:1207.0391v2, [math.AG] 5 Jan 2014.

[2] Francesca Acquistapace, Fabrizio Broglia, and Andreea Nicoara, A Nullstellensatz for Łojasiewicz ideals, Rev. Mat. Iberoam. 30 (2014), no. 4, 1479-1487, DOI 10.4171/RMI/822. MR.3293441 
[3] Edward Bierstone and Pierre D. Milman, Resolution of singularities in Denjoy-Carleman classes, Selecta Math. (N.S.) 10 (2004), no. 1, 1-28, DOI 10.1007/s00029-004-0327-0. MR2061220 (2005c:14074)

[4] Edward Bierstone, Pierre D. Milman, and Guillaume Valette, Arc-quasianalytic functions, Preprint. arXiv:1401.7683v1 [math.CV] 29 Jan 2014.

[5] Walter Rudin, Real and complex analysis, 3rd ed., McGraw-Hill Book Co., New York, 1987. MR.924157(88k:00002)

[6] Vincent Thilliez, On quasianalytic local rings, Expo. Math. 26 (2008), no. 1, 1-23, DOI 10.1016/j.exmath.2007.04.001. MR2384272(2009m:32007)

Dipartimento di Matematica, Università degli Studi di Pisa, Largo Bruno PonteCORVO, 5, 56127 Pisa, ItALY

E-mail address: acquistf@dm.unipi.it

Dipartimento di Matematica, Università degli Studi di Pisa, Largo Bruno PonteCORVo, 5, 56127 Pisa, Italy

E-mail address: broglia@dm.unipi.it

School of Mathematics, Trinity College, Dublin, College Green, Dublin 2, Ireland

E-mail address: anicoara@maths.tcd.ie 